\title{
La relación entre productor y consumidor en sistemas alimentarios locales: análisis de sus prácticas y narrativas
}

\author{
Eleni Papaoikonomou y Matias Ginieis \\ Universidad Rovira i Virgili, Tarragona \\ eleni.papaoikonomou@urv.cat, matias.ginieis@urv.cat
}

Resumen: Los sistemas alimentarios locales, conocidos como local food systems, ban crecido en popularidad en todo el mundo en los últimos años. Previa literatura en este campo ba puesto énfasis en el carácter contrahegemónico de estas iniciativas resaltando la re-conexión de los productores y los consumidores $y$, a su vez, se señala una objeción a la agricultura industrial que ofrece productos anónimos y desconectados de su productor y de su lugar de origen. Sin embargo, dichos discursos románticos no siempren se comprueban a través de la evidencia empirica. En este estudio realizamos una comparación empirica entre dos sistemas alimentarios locales: las denominadas Community Supported Agriculture en Manhattan, Nueva York y las Cooperativas de Consumo Responsable en Cataluña, España. En base a la utilización de una metodología cualitativa se identifican y se exploran las prácticas y narrativas de establecidas entre el productor y consumidor que también se definen por la orientación y raison d'être del sistema local.

Palabras clave: sistemas alimentarios locales; prácticas; narrativas; cualitativo.

Abstract: Local food systems have gained popularity over the last years. Previous research discusses the counter hegemonic potential of these initiatives placing emphasis on the reconnection of the producer and consumer of food and against the anonymous and disconnected production model offered by industrial agriculture. However, such romantic discourses do not always apply according to empirical evidence. In this paper we carry out an empirical comparison between two local food systems: the Community Supported Agriculture in Manhattan, New York and the the Responsible 
Consumption Cooperatives in Catalonia, Spain. Using a qualitative methodology we explore practices and narratives that surround the consumer-producer relationship, also defined by the orientation and raison d'être of the local food system.

Keywords: local food systems, practices, narratives. 


\section{Introducción y conceptualización}

El crecimiento del comercio mundial ha supuesto la especialización y la deslocalización de las producciones agrarias (Langreo Navarro, 2008). La industrialización y la globalización de la agricultura han recibido críticas con respecto a la concentración de poder en algunas pocas empresas multinacionales, al deterioro y el empobrecimiento de tejidos económicos y sociales locales, y a la falta de soberanía alimentaria de los países (Delgado, 2010; y Montagut y Vivas, 2012). En este contexto, se ha observado un rápido crecimiento en la tendencia al consumo de alimentos locales y en su adopción como una solución a los problemas de la agricultura intensiva mundial (DuPuis y Goodman, 2005; y Nie y Zepeda, 2011). Estas tendencias de consumo encajan con lo que Alonso (2005) denomina el nuevo consumidor, quien busca formas alternativas, saludables y sostenibles de consumo. Cabe aclarar que cuando se alude a la localización del suministro de alimentos, se señala que los alimentos deben ser consumidos lo más cerca posible de su punto de producción. Sin embargo, la literatura previa demuestra que el término local ha sido sujeto a numerosas interpretaciones, es decir, por consumo local puede entenderse el consumo de productos en el municipio, en la provincia, en la región o en el país del consumidor (Selfa y Qazi, 2005; Seyfang, 2006; y Feldmann y Hamm, 2015). Así pues, la tendencia a consumir alimentos en el ámbito local ha supuesto la afluencia de los movimientos de consumidores locales, denominados locavores (Rudy, 2012), y la aparición de un abanico de opciones para comprar y comer en negocios especializados en alimentos locales, como los mercados de agricultores locales, los restaurantes $\mathrm{km} 0^{1}$ o los sistemas alimentarios locales, donde grupos de consumidores compran directamente a los productores locales.

Asimismo, el interés social por esta tendencia ha propiciado que se realicen distintos trabajos empíricos que estudian las motivaciones de los consumidores locales y el funcionamiento de los sistemas alimentarios locales. Numerosos consumidores eligen consumir de forma local porque consideran que los productos locales son más frescos, saludables y de mayor calidad (Khan y Prior, 2010; y Feldmann y Hamm, 2015). Seyfang (2006) establece que también los consumidores locales tratan de apoyar la economía local o escogen esta forma de consumo porque es más sostenible, puesto que se reducen así las emisiones de carbono relacionadas con el transporte de alimentos en todo el mundo. Además, el consumo local de alimentos a menudo se presenta como un tipo de activismo alimentario basado en la ética que desafía a la hegemonía industrial, al sistema capitalista y a

1 Son restaurantes que utilizan exclusivamente productos locales y transmiten la filosofía del movimiento slow food (véase el siguiente enlace: $<$ http://slowfood.es/restaurantes-km0>). 
los sistemas agroalimentarios globalmente integrados (Hinrichs, 2003; DuPuis y Goodman, 2005; Seyfang, 2006; y Johnston, 2008). De esta manera, los sistemas alimentarios locales están enmarcados en beneficio de las ecologías locales y de las relaciones sociales locales (DuPuis y Goodman, 2005; y Jarosz 2008). Por lo tanto, la relocalización del consumo de alimentos tiene un gran potencial para construir un capital social en las cadenas alimentarias, que reconectan a los consumidores y a los productores de alimentos (Hinrichs, 2003; y Seyfang, 2006). Así, acortar la cadena de suministro reconecta al consumidor y al productor, y también aumenta el placer y el valor de uso para el consumidor (Adams y Raisborough, 2008).

No obstante, a pesar de que las narrativas románticas del consumo local se han aplicado asiduamente (Pratt, 2007; y Dowler y otros, 2009), a veces los sistemas alimentarios locales pueden llegar a emplear técnicas agrícolas industrializadas o incluso a explotar a sus trabajadores (Allen y otros, 2003; Jarosz, 2008; Nost, 2014; y O'Neill, 2014). Asimismo, aunque estos sistemas se posicionan en contra de los alimentos anónimos y desconectados de su producción (Murdoch, 2000; y Selfa y Qazi, 2005), no consiguen reconectar al consumidor con el productor a través de una relación directa y personal (DeLind, 1999; Allen y otros, 2003; Selfa y Qazi, 2005; y Ostrom, 2007). En este sentido, cabe destacar que estas iniciativas locales pueden diferir drásticamente dependiendo de su grado de semejanza con el sistema alimentario industrial, de la relación que se establece entre el productor y el consumidor, y de su organización y gobierno (Lamine, 2005; y Feagan y Henderson, 2009). Así pues, frecuentemente se le confiere legitimidad a la práctica de consumo local por su «moralidad»y su potencial transformador. El consumo local puede comodificarse de forma romántica (Varul, 2008) a través de la manipulación de su significado. Esta práctica de consumo emerge como símbolo de un sistema de producción-consumo sostenible, «moral», alternativo y auténtico, utilizado por el consumidor para su construcción como sujeto (Varul, 2008), para la producción de un «yo moral» socialmente reconocible (Adams y Raisborough, 2008). Asimismo, el consumo local puede plantearse como una opción transformadora si desafía, en cierta manera, el sistema de producción-consumo dominante en lugar de utilizarse meramente para propósitos de identificación de los individuos.

El presente trabajo pretende seguir esclareciendo la relación construida entre los consumidores y los productores a través del estudio de dos sistemas alimentarios locales: la agricultura sostenida por la comunidad (o community supported agriculture; en adelante, CSA) de Nueva York y la cooperativas de consumo responsable (en adelante, CCR) de Cataluña. La literatura revisada hasta la actua- 
lidad indica que se posee una noción limitada de cómo se establece el vínculo entre consumidores y productores en las cadenas alimentarias locales, por lo que se trata de comprender en profundidad esta relación mediante la exploración de las prácticas y las narrativas construidas a su alrededor. Según Reckwitz (2002), las prácticas son modos de comportamiento rutinizados, mientras que Warde (2005) sugiere que las prácticas consisten en hechos y narrativas, en la actividad práctica y en su representación discursiva. En términos de análisis, las prácticas se encuentran entre lo social y lo individual (Warde, 2005), enfatizando el carácter colectivo del consumo. Tras haber realizado una comparación constante entre dos grupos de consumo local ubicados en diferentes contextos culturales, los resultados obtenidos en el estudio desarrollado identifican diferentes tipos de prácticas y narrativas. Tanto las CSA como las CCR fueron seleccionadas para este estudio porque a diferencia de los mercados de agricultores y de las tiendas de alimentos locales (DeLind, 1999; y Feagan, 2007), poseen el potencial necesario para establecer relaciones significativas entre los consumidores y los productores, puesto que requieren cierto nivel de compromiso y colaboración por ambas partes (Kneafsey y otros, 2008).

Por lo tanto, el presente trabajo se estructura de la siguiente manera. En primer lugar, se explicará detalladamente la metodología empleada. En segundo lugar, se expondrán las principales características de las CSA y de las CCR. Y por último, se presentarán y discutirán las principales aportaciones que proporciona la comparación de los dos sistemas alimentarios locales estudiados.

\section{Metodología del estudio}

Este trabajo presentará los resultados de dos estudios cualitativos sobre grupos de consumidores de alimentos locales. En el primero de ellos se analizaron las CCR de la región española de Cataluña, mientras que el segundo estudio se centró en las CSA urbanas de la ciudad de Nueva York. En ambos casos se adoptó un enfoque cualitativo interpretativo para facilitar un análisis profundo, así como una comprensión holística de la relación que se establece entre los consumidores y los productores de los grupos de alimentos locales.

La metodología adoptada en esta investigación ha sido la teoría fundamentada en datos (grounded theory). De acuerdo con Corbin y Strauss (1990), se reconoce la «sensibilidad teórica» de los investigadores, que les posibilita identificar conceptos relevantes. Esta metodología le permite al investigador reflexionar sobre los datos recopilados, ya que se recogen y examinan a través de un análisis constante, que lleva a nuevas rondas de recolección y análisis de datos hasta la saturación teórica. Así pues, se ha adoptado un muestreo teórico y heterogéneo 
para verificar si nuevas variables eran relevantes; por ejemplo, la edad de los grupos de consumo estudiados (Patton, 2002). Además, esta metodología permite la aparición de códigos centrados en los participantes (participant-centered) en lugar de categorías impulsadas por los investigadores (Corbin y Strauss 1990; y Glaser y Strauss, 1967). Mediante la adopción de un enfoque constructivista, se acepta el carácter performativo de la investigación (Heiskanen, 2005; y Charmaz, 2006), teniendo en cuenta que los resultados son «construcciones conjuntas de conocimiento» producidas por la interacción entre las narrativas de los participantes y sus interpretaciones (Mauthner y Doucet, 2003: 424).

A continuación, se procederá a explicar brevemente el funcionamiento de los dos sistemas estudiados y los aspectos específicos de la metodología adoptada en cada uno de estos análisis.

\subsection{El estudio sobre las CCR (Cataluña)}

Las CCR son grupos de consumo local cuyo principal proyecto implica la compra colectiva de productos de acuerdo con los criterios éticos establecidos por el grupo. En estos criterios se prioriza: a) la preferencia de pequeños productores locales, $b$ ) la elusión de los intermediarios, ya que se busca una relación directa con el productor, y c) la compra de productos ecológicos que se hayan generado bajo condiciones laborales justas. A menudo, la elección de un productor significa conocer en profundidad su forma de trabajar y su empresa. Cabe destacar que los consumidores no eligen los productos que van a consumir, sino que reciben los productos de temporada que el agricultor ha cultivado. Además, los estatutos de todas las CCR participantes establecen claramente como núcleo de su existencia la importancia del autogobierno, de la toma de decisiones democrática y de la igualdad de participación a través de la distribución del trabajo y de las responsabilidades.

Para seleccionar las CCR que participaron en el estudio se utilizaron como marcos de muestreo inicial una revista catalana de consumo responsable y los directorios de cooperativas. Con respecto a los métodos de investigación, se llevó a cabo una investigación multimétodo, es decir, se combinaron cuatro técnicas de recolección de datos: observación, entrevistas, grupos de discusión y análisis de documentos. El trabajo de campo comenzó con la técnica de observación (tanto online como offline) que permitió a los investigadores establecer un rapport con los miembros de la cooperativa (Patton, 2002). La técnica de observación incluyó varias visitas a diferentes CCR en Cataluña (en su mayoría urbanas y ubicadas en Barcelona) y la asistencia a eventos organizados por estas, como la reunión anual Repera, que reúne a agricultores, productores y consumidores. En algunos casos, 
el investigador simplemente actuó como mero espectador, mientras que en otros, adoptó un papel de observador participante. Cabe mencionar que, en la mayoría de los casos, las CCR tienen su propio espacio permanente, que otras entidades alquilan $u$ ofrecen. La existencia de un espacio permanente es importante, ya que a veces es imprescindible para la organización de eventos, asambleas, seminarios, etc. Asimismo, se llevaron a cabo cuatro grupos de discusión (32 participantes), cuyas sesiones duraron de 90 a 120 minutos, y nueve entrevistas en profundidad con una duración de 40 a 120 minutos cada una. Las entrevistas se basaron en preguntas abiertas, puesto que incitaban a la aparición de nuevos temas y datos (Patton, 2002). Por último, se utilizó el análisis de documentos como técnica complementaria, que consistió en el análisis de los estatutos y de otros documentos, así como de la revista $E l$ Troc (disponible de forma gratuita en internet), que los miembros de las CCR escriben y editan ellos mismos.

\subsection{El estudio sobre las CSA (Nueva York)}

El concepto de agricultura sostenida por la comunidad (CSA) surgió del sistema teikei japonés, que se basa en un tipo de coasociación entre agricultores y consumidores para garantizar la seguridad alimentaria y proporcionar beneficios para ambas partes (Feagan y Henderson, 2009). A mediados de la década de los ochenta, este movimiento se extendió a Europa y a los Estados Unidos, donde inicialmente representaba un nicho en el mercado, aunque actualmente su popularidad ha aumentado de forma notable (Perez y otros, 2003). En una CSA, un agricultor de la zona, que a menudo trabaja con métodos de producción orgánicos, y los residentes locales se organizan para establecer una red de comercialización entre ellos. Los consumidores compran y pagan de antemano la producción de la granja local y reciben una caja con productos agrícolas de temporada cada semana. Normalmente, en Estados Unidos hay una o dos temporadas: la de primavera-verano y, a veces, la de otoño-invierno. Como en las CCR de Cataluña, los consumidores de las CSA no eligen los productos que se cultivan. Además, está establecido que en caso de una catástrofe natural u otros problemas similares, los consumidores no podrán solicitar el reembolso de lo ya pagado. Compartir el riesgo es fundamental para el modelo de CSA, y según Hinrichs (2000), esta función de riesgo compartido y del pago por adelantado hace hincapié en las «relaciones sociales, ambientales y culturales más amplias», más allá de las consideraciones del mercado o del producto en sí (Feagan y Henderson, 2009: 205).

Las CSA fueron seleccionadas a través de los directorios en línea y del sitio web Just Food (<http://www.justfood.org/csa>). Las principales técnicas de recolección de datos fueron las siguientes: entrevistas (15) y observación. Se 
entrevistaron a trece miembros de las CSA, que representaban a trece grupos de consumidores diferentes, y a dos productores que abastecen a diferentes CSA en la isla de Manhattan. En menor medida también se utilizó la técnica de análisis de documentos, ya que los miembros de las CSA proporcionaron documentación, como boletines e informes, entre otros documentos. Una CSA, por ejemplo, facilitó un informe desarrollado por el propio grupo sobre la experiencia de sus miembros.

La observación se llevó a cabo durante los días de distribución de las CSA con un procedimiento similar al seguido durante el primer estudio. Aunque en otros modelos de CSA, los miembros tienen que ir a la granja a recoger su caja (Perez y otros, 2003; Thompson y Coskuner-Balli, 2007; y Nost, 2014), en este el agricultor entrega el producto en un lugar designado en la ciudad y en unos días de distribución específicos (por ejemplo, todos los miércoles del mes). Las CSA raramente tienen su propio espacio particular $y$, por ello, utilizan una gran variedad de lugares para la entrega de sus productos, como iglesias, jardines o centros comunitarios. El lugar de entrega se decide en función de la disponibilidad y la conveniencia del espacio y de los miembros.

El uso de diferentes técnicas de investigación facilitó la triangulación de datos. En cuanto a la interpretación de estos datos y a su análisis se siguieron los principios básicos de la teoría fundamentada en datos, por lo que inicialmente se llevó a cabo una codificación abierta (open coding) y posteriormente, una codificación axial y selectiva (axial and selective coding). Con este análisis, se buscó una comprensión holística de los datos cualitativos a través de un proceso iterativo de interpretación y reinterpretación de los datos, estableciendo relaciones conceptuales entre los códigos emergentes (Glaser y Strauss, 1967). Bajo este enfoque, los significados personales y las narrativas se entendieron dentro de un sistema más amplio de supuestos culturales, de conocimiento cultural y de socialización. Para organizar y gestionar el gran volumen de datos generados y para aumentar su transparencia, se utilizó el software NVivo, que facilitó el proceso de análisis.

Los datos principales de estos dos estudios se describirán en la siguiente sección y se resumirán en la figura 1. 


\section{Resultados}

\subsection{Sistemas alimentarios locales: la relación entre productor $y$ consumidor}

En la sección anterior se han descrito las CSA y las CCR como iniciativas locales que fomentan los ciclos cortos de producción-consumo a través de la eliminación de cualquier intermediario. La elección de productores locales permite recuperar una relación directa entre el productor y el consumidor, debido a que los sistemas modernos de intermediación hacen este proceso altamente no transparente (Cook, 2004). Dowler y otros (2009) explicaron que esta desconexión en el sistema alimentario ha creado la necesidad de volver a conectar sus diferentes elementos: el consumidor, el productor y la naturaleza.

En este apartado, se presentarán las principales conclusiones sobre cómo se articula la relación y la reconexión de los productores y los consumidores a través de sus narrativas y las prácticas establecidas en los dos grupos explorados. De acuerdo con Dowler y otros (2009), esta relación se concibe como un proceso dinámico. Se han identificado dos tipos de prácticas y narrativas: de compartir y de negociar — las cuáles se explicarán más adelante-. Los resultados obtenidos permiten comprender todas las dimensiones en las que se establece la relación entre los consumidores y los productores y ver hasta qué punto la romantización del consumo local se corresponde realmente con la reconexión de los diferentes elementos del sistema alimentario. Se presentarán ejemplos específicos de eventos y rituales en los que el productor y el consumidor participan. Además, el análisis llevado a cabo mostrará la interrelación entre las prácticas establecidas, las narrativas, los esquemas de organización y el gobierno de los grupos locales, y cómo todos estos definen la orientación y el carácter transformador del grupo.

\subsubsection{Prácticas y narrativas de compartir}

Investigaciones anteriores sobre los sistemas alimentarios locales han descrito los eventos, los rituales y las prácticas que tienen como objetivo reconectar al productor y al consumidor (Thompson y Coskuner-Balli, 2007; Feagan y Henderson, 2009; y Nost, 2014). Los resultados empíricos obtenidos en este estudio confirman la existencia de estos rituales y prácticas. Los agricultores y los consumidores se reúnen durante la entrega de los productos, pero también organizan comidas informales, visitas a granjas, seminarios o, incluso, conferencias anuales. Por ejemplo, la Repera es una conferencia que reúne a miembros de las CCR catalanas y a agricultores locales. Se inició en 2008 y se celebra anualmente en diferentes localizaciones. Tiene una duración de un fin de semana e incluye varie- 
dad de eventos. En la $6 .^{a}$ edición de la Repera, el programa consistió en talleres sobre cómo crear una CCR; en debates sobre cuál debe ser la relación entre los consumidores y los productores, y sobre el futuro de la agroecología; y en una comida con música.

Figura 1. Principales resultados de los dos estudios efectuados.

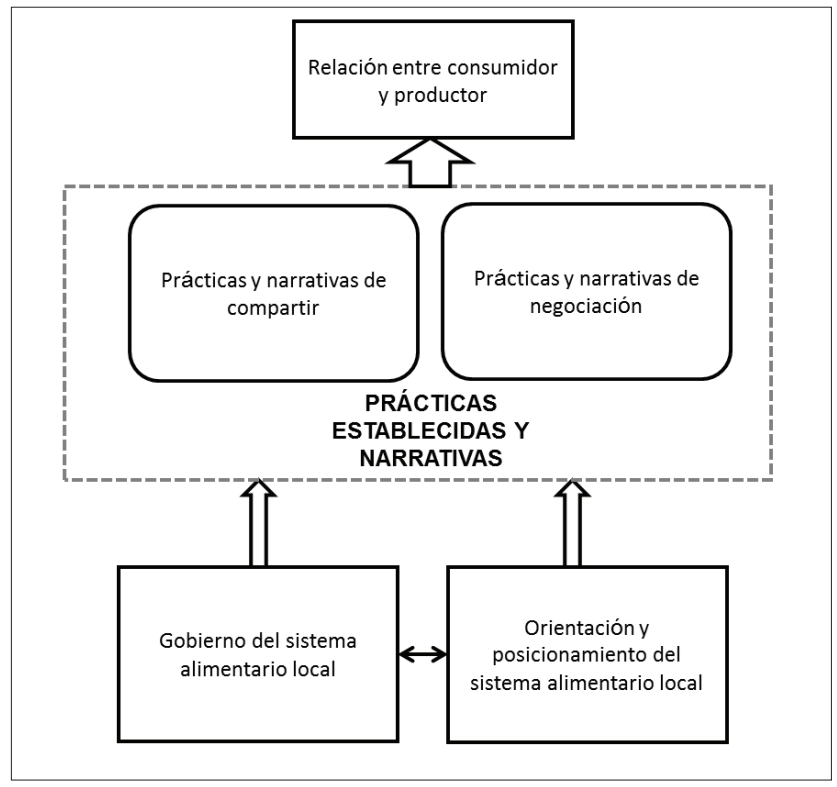

Fuente: elaboración propia.

Después de esta conferencia, los miembros de las CCR publican información y fotos en su blog. Esta es una práctica representacional que aumenta la visibilidad de estos eventos. El anuncio de los organizadores de la $6{ }^{a}$ edición de la Repera decía lo siguiente:

Como algunos ya saben, la Repera son conferencias anuales donde nos encontramos, celebramos y fortalecemos nuestras identidades agroecológicas. Por lo tanto, hacemos un llamado a los grupos de consumidores, agricultores y otros productores $y$ a todos los que quieran aprender sobre esta realidad. Cada año celebramos la Repera en una ciudad diferente, así llegamos a conocer mejor nuestra tierra $y$ a su gente.

Estas conferencias señalan la desconexión entre los diferentes actores y la voluntad de actuar sobre ella a través de la promulgación de estos rituales anuales (Kneafsey y otros, 2008; y Dowler y otros, 2009). De este modo, se crea una red más amplia, que conecta a los agricultores y a las microcomunidades de consu- 
midores. Otros rituales bien establecidos, tanto en las CCR como en las CSA, incluyen visitas a las fincas de los productores. La siguiente cita describe la rutina seguida durante una visita de una CSA a la finca del productor:

Éramos siete y nos fuimos hasta allí. Él [el productor] nos dividió a todos en dos grupos. Lo primero que hicimos fue ver las colmenas. Recibimos miel de allí, así que fuimos a verlo. Luego fuimos a ver los pollos, y algunas personas fueron a plantar semillas, mientras otros se encargaban de los tomates, y otro plantaba hierbas. Luego fuimos a la casa del granjero y su esposa nos hizo unos bocadillos y hablamos de lo que esperábamos de la temporada y cosas así, y luego nos volvimos a casa. Eran cuatro horas y media en coche (Alison).

Aquí Alison describe una visita estándar de una CSA a una granja. Sin embargo, aunque estas visitas son rituales bien establecidos para «educar» a los consumidores y reconectarlos con el productor, solo 7 de aproximadamente 100 miembros fueron a la granja. La participación limitada por parte de los miembros se debe principalmente a la falta de tiempo y a que las granjas se encuentran lejos de la ciudad. Para la gran mayoría, el contacto con el productor se limita al día de entrega de productos o ni siquiera entonces, dado que el productor se marcha cuando entrega sus productos. Así pues, si la relación establecida entre el consumidor y el productor es limitada o inexistente, ¿cuál es el significado del consumo local para los miembros de la CSA? Al igual que en anteriores estudios empíricos (Khan y Prior, 2010; y Feldmann y Hamm, 2015) la frescura del producto y la calidad se enfatizan como las principales motivaciones de los miembros de las CSA, mientras que la noción de consumo local no se traduce en una relación real con el productor, sino en una relación imaginaria (DeLind, 1999; y Nost, 2014). Elisa, un miembro de una CSA, no siente que forma parte de una comunidad real, sino de una comunidad imaginaria por varios factores: el tamaño de la CSA, que hace que sea menos personal; los horarios incompatibles de sus miembros; $y$ la falta de tiempo. Sin embargo, aunque no existe el sentido de comunidad ni la creación de una identidad social o capital social, sus miembros parecen utilizar su pertenencia a la CSA para propósitos de identificación personal.

Es difícil porque en Nueva York todo el mundo está muy ocupado y no tenemos mucho tiempo. Por lo tanto, yo no lo llamaría como una comunidad. Por desgracia, me siento como, no sé... La gente solo quiere recoger su caja, pero siento que soy parte de esta comunidad invisible y eso es suficiente para mí. Desearía que fuera más pequeña y todos se conocieran, pero ese no es el caso (Elisa).

Estos relatos encajan con una orientación instrumental de la CSA que se asemeja a una transacción mercantil (Feagan y Henderson, 2009). 
En el caso de las CCR, estas priorizan más la relación directa con el productor y la participación activa de todos sus miembros. Prácticas y normas como un número máximo de miembros, asambleas y visitas frecuentes a los productores intentan crear pequeños grupos en los que se elimina el anonimato entre los consumidores y los productores. Cabe mencionar el caso de una CCR recién establecida en la que había tensiones entre sus miembros por los criterios de compra que querían establecerse. Algunos miembros querían, básicamente, que los productos fueran ecológicos, aunque no locales; es decir, estaban dispuestos a aceptar productos de intermediarios ecológicos. Sin embargo, otros miembros, como Manolo, se oponían a este criterio porque consideraban que esto generaba la misma dinámica de consumo que pretendía evitarse.

Si se acepta lo que sea, entonces es lo mismo. La misma dinámica de consumo. Lo único que no se consumen productos con pesticidas. No cambiamos nada (Manolo).

Manolo, entre otros miembros, cree en el potencial transformador del grupo. Si en la CCR se ofrecen solamente productos frescos y orgánicos como en cualquier megatienda orgánica, entonces no se desafía realmente al modelo de agricultura industrial y a la mercantilización de la producción (Johnston, 2008). Esta desmercantilización se relaciona directamente con el tipo de relación que se establece entre productores y consumidores, insistiendo en la atención a la relación social establecida en lugar de concebir el producto como commodity (Feagan, 2007). De hecho, algunos miembros hacen hincapié en su cercanía con los productores, a los que a menudo llaman amigos. Para Manolo, si la relación establecida entre los productores y los consumidores se asemeja a las transacciones mercantilizadas, se limitan las aspiraciones antihegemónicas de su grupo de consumo local.

Volviendo al caso de las CSA, hay una CSA que es una excepción, puesto que ha estado trabajando durante más de 17 años con los mismos agricultores. Entre sus prácticas de reconexión entre los productores y los consumidores se encuentra una gran reunión en la granja cada otoño, en la que todos comparten una comida. Además, los miembros de esta CSA saben mucho acerca de los agricultores con los que trabajan y sus productos.

[... Tenía cuatro niños pequeños en casa y era una apasionada de la agricultura ecológica, pero no quería ir al mercado de los agricultores porque eso significaba que pasaría tiempo lejos de los niños. Sin embargo, con la CSA podía hacer la entrega una vez por semana: conducía el camión hasta Nueva York y volvía a tiempo para cuando los niños llegaban a casa de la escuela. Ahora, él [el agricultor que hace la entrega] se ha especializado en gestión agrícola y se ha unido 
oficialmente a la granja. Fue la visión de Jane [seudónimo de la madre] y su pasión lo que ha hecho que esto sea posible.

[... J Jane es una mujer increíble. Cuando empezó, todos esperaban que fracasase, todos pensaron que era tonta. No obstante, se adelantó a su tiempo y ahora trabaja con 16 o 18 CSA (Rose).

Como en otros estudios (Thompson y Coskuner-Balli, 2007), se aprecia una visión romántica del agricultor, que se concibe como un artesano noble, como un luchador.

En las CCR, hay narrativas similares, en las que los productores se presentan como «guardianes de la ecología, la tradición agrícola y variedades de semillas locales» o como personas que «no quieren ser ricos, que solo creen en lo que están haciendo» (revista El Troc). Tanto los miembros de las CCR como los productores trabajan conjuntamente para proteger las variedades locales de semillas que forman, en definitiva, parte de su identidad cultural común. Este objetivo podría enmarcarse como una resistencia a la hegemonía global de homogeneización cultural de variedades y de las tradiciones alimentarias (Feagan, 2007). Por lo tanto, localismo, en este caso, se refiere a la preservación de la cultura y de las tradiciones, más allá del acceso a la calidad y a los alimentos ecológicos.

\subsubsection{Prácticas y narrativas de negociar}

Un tema que ha recibido escasa atención en la literatura es el proceso de negociación que tiene lugar entre los miembros de los sistemas alimentarios locales y los productores con los que trabajan. En el caso de las CCR, los miembros afirman mantener una relación abierta en la que ambas partes participan por igual. Como grupo, todos los miembros tienen que asumir un papel activo. Sus responsabilidades conllevan el pago de un capital inicial (que se devuelve si el miembro decide abandonar la cooperativa), una cuantía anual para gastos de funcionamiento (por ejemplo, el alquiler y otros servicios públicos) y el coste semanal de los productos adquiridos. Además, deben participar en la toma de decisiones y en el funcionamiento de la cooperativa a través de asambleas y comités de trabajo. Como caso representativo, cabe destacar una CCR que tiene cuatro grupos de trabajo diferentes: la comisión de compras, a cargo de encontrar y negociar con los productores (dónde se hacen los pedidos, las visitas a los productores, etc.); la comisión de administración, a cargo de las tareas administrativas (pago de alquiler y otros gastos); la comisión de comunicación, a cargo de las relaciones exteriores con los medios de comunicación, con otras cooperativas, con los investigadores, etc; y la comisión de actividades, encargada de la organización de seminarios, talleres, etc. 
Los miembros de la CCR deben colaborar en uno de estos comités e ir rotando durante su participación, aunque también se han observado free riders.

En las CCR se hace hincapié en que el flujo de información dentro del grupo debe ser centralizado, es decir, que los criterios de compra, así como el resto de decisiones deben establecerse a través del consenso de todos los miembros. La negociación directa con el productor permite conseguir más transparencia y control sobre el proceso productivo de manera más real. Uno de los miembros encargados de esta negociación es Marta, que es ingeniera agrícola y pertenece a la comisión de compras de su CCR. Además, dada su experiencia profesional, incluso dialoga con los productores sobre sus métodos de cultivo en lugar de limitarse a una «promesa de calidad» (Lamine, 2005). Esto también conduce a un mayor empoderamiento colectivo, ya que los consumidores negocian y deciden como grupo en vez de individualmente, lo que crea una nueva alternativa de consumo y participación social (Johnston, 2008).

En la revista El Troc, varios artículos describen las visitas de los miembros de las CCR a sus productores y dan información sobre ellos, su historia, sus granjas y sus productos. Estas formas de obrar refuerzan la autenticidad de los alimentos producidos y consumidos (Varul, 2008). No obstante, si el productor no cumple con los requisitos que establece la cooperativa su colaboración puede terminarse, como sucedió en el siguiente caso.

Bueno, nuestro proveedor de cebollas y ajos aquí, en Falset, se jubiló y tuvimos a otro, pero lo que producía no era suficiente. Por lo tanto, fuimos a un distribuidor en el que confiamos mucho para que nos lo trajera [el producto], pero

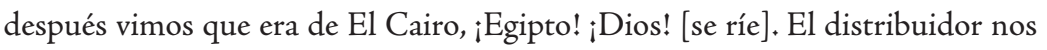
dijo: «Pero si es cebolla ecológica!». No podemos permitir como cooperativa, incluso si es ecológica, una cebolla de El Cairo; y la habíamos consumido ya durante 15 días. Llamamos al distribuidor y le dijimos: «iAquí no lo queremos, y si no tienes, pues nada más!» (Carmen).

Carmen explica que cuando descubrieron que el producto no era local, la colaboración se terminó inmediatamente, a pesar de que al principio confiaban en ese distribuidor. Los participantes de las CCR dejan claro y enfatizan constantemente su deseo de recuperar la transparencia de lo que compran: de dónde y de quién provienen. Los participantes, junto con los productores y proveedores, que entienden sus criterios y su ideología, tratan de construir sus opciones de consumo activa y colectivamente. A veces, algunas CCR, incluso, organizan la propia producción de los productos que necesitan, como la pasta de dientes, y/o alquilan tierras para cultivar algunas verduras por cuenta propia. 
En cambio, el estudio de las CSA de Nueva York muestra que aunque existe cierta carga de trabajo para sus miembros, esta es menor que en las CCR. Por ejemplo, una de las CSA colgó esto en su sitio webः «Cada miembro debe trabajar un turno de 2 horas en la entrega de productos a lo largo de la temporada». Sin embargo, en la mayoría de las CSA de nuestro estudio, sus miembros no parecen participar en un proceso de negociación con el productor y tienden a entrar en un acuerdo con términos predefinidos.

Investigador: - ¿Cómo encontró al agricultor?

CSA: - Just Food nos lo encontró.

Investigador: - ¿Estáis en contacto con el agricultor? ¿Lo veis o vais a la granja?

CSA: - Solo durante la distribución. Nos han invitado a la granja, pero hasta ahora no hemos ido.

Investigador: - ¿Tenéis un número máximo de miembros?

CSA: - Se nos dijo que el agricultor quería entre unos 25 y 30 miembros.

Investigador: - Ok. ¿Alguna vez le habéis dicho al agricultor «no nos gustan estos vegetales»o «queremos más cantidad o menos cantidad»?

CSA: - Formar parte de la CSA es aceptar lo que la granja vende.

Como ya hemos mencionado, ninguno de estos dos grupos decide qué consumirá, por lo que la caja semanal de verduras y/o frutas es una sorpresa. No obstante, existen diferencias entre los dos sistemas locales. En las CCR catalanas, los propios miembros deciden cuántas personas se necesitan para asegurar el autogobierno de su grupo y su capacidad para tomar decisiones, y lo hacen por consenso en lugar de por votaciones. Ellos deciden con qué productores trabajarán y llegan a acuerdos con estos. Sin embargo, este no es el caso para todas las CSA de Nueva York, donde el tamaño del grupo puede llegar a depender de los agricultores. Además, parece que en las CSA de este estudio, los miembros no entran en un proceso de negociación y diálogo. Aunque Kneafsey y otros (2008) remarcan la importancia de las relaciones sociales en las CSA para reconstruir la confianza entre el agricultor y los consumidores, se ha observado que la selección de los agricultores no la hacen en grupo. La entrevista a una empleada de Just Food proporciona una visión mucho más completa sobre cómo se escoge a los agricultores.

A veces las personas vienen y nos dicen: «Queremos una CSA en nuestro barrio o en nuestro lugar de trabajo». Les ayudamos a empezar. Les hacemos llegar a los agricultores una solicitud y les decimos que buscamos algunas cosas. En primer lugar, tienen que obtener una certificación de cultivo orgánico o algo parecido. Tienen que estar a menos de $250 \mathrm{~km}$ de la ciudad y ser fincas diversificadas. Es bueno que tengan experiencia con otras CSA, que hayan trabajado en 
una granja de una CSA. Si no, les ayudamos. Es necesario que tengan un camión de confianza y suficiente mano de obra y tierras, este tipo de cosas. Por lo tanto, llenan la solicitud y un comité asesor agricultor, constituido por algunos agricultores de las CSA, por el personal de Just Food y por especialistas en agricultura, la revisa. Comprobamos la solicitud y cualquier otra cosa que parezca extraña o problemática, y luego visitamos la finca para verla por nuestra cuenta y hablar con el agricultor. Nos convertimos en un intermediario que pone en contacto a agricultores y a consumidores. A veces es solo la logística: ¿Qué día de la semana lo pueden hacer?, ‘cuántos miembros quieren o necesitan?, ¿cómo de grande debe ser el grupo?, ¿están cerca de la ciudad? En fin, debemos asegurarnos de que sea un buen acuerdo (Paula).

En nombre de los miembros de las CSA, Just Food comprueba la autenticidad del productor y sus métodos. Ellos se ponen en contacto con los productores y deciden qué agricultor se ajusta mejor a cada CSA. Por ejemplo, en el caso de una CSA de solo solteros, que necesitan pequeñas cantidades de productos, Just Food busca qué agricultor les puede abastecer. La entidad también ayuda a sus miembros a empezar con la organización de la CSA. Organizan seminarios e, incluso, proporcionan a algunas CSA visibilidad, sobre todo a aquellas que no tienen una página web propia y solamente aparecen en el sitio web de Just Food. A diferencia de las CCR catalanas, este sistema muestra un alto nivel de profesionalización, puesto que participan expertos en agricultura y en procesos formales de selección centralizados. Just Food facilita claramente la expansión del modelo de CSA, ya que, como menciona el miembro de una CSA, «no tenemos que reinventar la rueda». Sin embargo, los participantes de las CSA también afirman lo siguiente: «Realmente dependemos de Just Food» y «no tenemos mucha autonomía». Se ha observado que sus negociaciones con el agricultor son limitadas y entran en acuerdos predefinidos con pocas decisiones por tomar. Un ejemplo que ilustra esta aseveración es que el criterio de lo que se considera local lo define Just Food (máximo unas 250 millas alejadas de la ciudad).

Él [el productor] nos da un precio a principios de la temporada y tiene un sistema en el que si uno se registra temprano obtiene descuento. Si se inscribe después de la fecha límite, se paga la totalidad del importe. Así funciona, de esta manera. Nos enteramos el lunes de lo que recibiremos en la entrega de la semana (Rose).

En ambos estudios, hay CCR y CSA con diferentes grados de complejidad. En algunos grupos, el suministro se limita a verduras y en otros grupos, la variedad de productos es mayor: frutas, carnes, pan, huevos, mermeladas, etc. En estos casos, las CCR negocian directamente con diferentes proveedores y así, estable- 
cen redes mayores y más complejas. Sin embargo, las CSA de nuestro estudio suelen utilizar a su agricultor como intermediario, que es quien negocia con los productores que conoce para ampliar el surtido de productos.

Investigador: - Así que, básicamente, ¿̇trabajáis con una granja grande que ofrece la mayoría de los productos?

CSA: - Sí. Esa es la norma en los Estados Unidos. El agricultor es la principal fuente de la CSA. Existen otros productores, pero sus productos vienen a través de nuestro agricultor. Los productos se entregan todos juntos a pesar de que el agricultor colabora con un gran número de proveedores.

El sitio web de consumo local <http://www.harvest.org> confirma que la última tendencia en las CSA es ofrecer un surtido más variado de productos: desde lácteos hasta flores. Así, frecuentemente, el agricultor de las CSA se alía con otros productores. En este caso, la tarea de autenticación se transfiere desde la entidad de Just Food al agricultor. Por el contrario, para los miembros de las CCR, es imprescindible participar activamente en el proceso de autenticación de los productos y de los productores: ¿Es el producto realmente local, saludable, justo y ecológico? Aquí, curiosamente, cabe destacar que ambos grupos hacen un uso limitado de los certificados oficiales de productos orgánicos para evaluar la calidad de los alimentos. En el caso de las CSA, los agricultores a menudo no tienen la certificación orgánica, ya que es un proceso caro y complicado. En su lugar, Just Food y sus expertos comprueban sus métodos. Como dice Paula: «Yo sé que George [seudónimo] no está certificado, quiero decir, que él está por encima de eso». Esto demuestra que la confianza en el productor puede sustituir o, incluso, superar los sistemas y las etiquetas de certificación de productos orgánicos oficiales. En cambio, en el caso de las CCR, sus miembros desafían abiertamente al sistema de certificación existente en España y consideran crear sus propias normas de calidad en lo que se refiere a la producción orgánica (Gertz, 2005). Los miembros de las CCR, en general, desconfían de los mecanismos implementados por el Gobierno y las administraciones.

Mira, en el CCPAE [Consejo Catalán de la Producción Agraria Ecológica, <http://www.ccpae.org>] no confiamos. Según este consejo, podemos tolerar un $0,9 \%$ de productos modificados genéticamente en los ingredientes. ¡Pero no lo hacemos! ${ }_{¡}$ No lo toleramos en absoluto! (Carmen).

Así que aunque ambas comunidades, las CSA y las CCR, coinciden en no hacer siempre uso de certificados oficiales y de etiquetas orgánicas, su razonamiento es diferente. Las CSA estadounidenses transfieren esta tarea a Just Food o al propio agricultor, quien les da las garantías y la confianza que necesitan. En cambio, las CCR catalanas mantienen una relación abierta y directa con sus productores 
y confían en ellos, a pesar de que participan de una manera más activa en los controles de calidad. Este último resultado también se relaciona con una mayor eficacia colectiva, percibida por parte de las CCR, quienes no parecen centrarse solamente en las normas de calidad que utilizan, sino que también se involucran en asuntos que son de interés para el grupo y que son importantes para lograr el cambio social deseado como ciudadanos y consumidores (Micheletti y Stolle, 2012).

\section{Conclusión}

Este trabajo se ha centrado en el estudio de estos dos sistemas alimentarios locales: las CSA estadounidenses y las CCR catalanas. Se han examinado estas iniciativas en el nivel micro y el análisis efectuado se ha centrado en el vínculo que se establece entre los consumidores y los productores, y en las prácticas y narrativas que se implantan en torno a esta relación. Principalmente, se han identificado prácticas y narrativas orientadas a compartir, y prácticas y narrativas sobre procesos de negociación. El presente estudio ha ahondado en la relación multidimensional que se crea entre los dos actores participantes en este tipo de sistemas: los productores y los consumidores. La relación entre consumidor y productor es clave en la comodificación romántica del consumo local (Varul, 2008), pero es importante entender las rutinas y los comportamientos colectivos que construyen este vínculo en el ámbito práctico. La comparación constante entre dos sistemas diferentes proporciona evidencia empírica sobre la heterogeneidad de los sistemas alimentarios locales en términos de su capacidad transformadora, sus aspiraciones y su orientación (Nost, 2014); un pluriuniverso social en el ámbito del consumo local (Alonso, 2005).

Las iniciativas elegidas para esta investigación se ubican en diferentes contextos culturales, aunque presentan similitudes, como la «caja de sorpresas»y la incertidumbre sobre la gama de productos (Lamine, 2005). Las diferencias importantes se encuentran en su administración, organización y posicionamiento ideológico. Por ejemplo, las CCR de Cataluña parece que intentan establecer un stakeholder, es decir, grupos con poder real o potencial para influir en las decisiones, a través de la creación de una red de consumidores y productores en el ámbito local, con lo que se busca un mayor control y transparencia en el consumo de alimentos y en su producción. Además, quieren contribuir al cambio social y, por ello, apuestan por el potencial transformador de sus grupos. Para ello, sus principios de funcionamiento, criterios de compra colectiva, prácticas y narrativas indican una dimensión más política de sus grupos, más allá del acceso a productos locales, frescos y de calidad. Algunos ejemplos de estas prácticas son la 
importancia de la autogestión, la creación de comisiones de trabajo y las frecuentes visitas a los productores, que requieren la participación activa de los consumidores en su red agroalimentaria. A menudo los productores y los consumidores se representan como los actores principales en la red agroalimentaria local, que trabajan conjuntamente para la protección del medioambiente; la preservación de las tradiciones agrícolas, las semillas y las variedades locales; y el mantenimiento de la identidad cultural común. Esto proporciona una idea más completa de lo que representa lo local para los miembros de las CCR.

En cambio, la mayoría de CSA de este estudio apuesta por los productos locales y frescos y por un contacto directo con el productor, en contra del anonimato del mercado. Cabe destacarse la existencia de la institución Just Food como la entidad gatekeeper en Nueva York, que facilita la aparición y el establecimiento de nuevas CSA en la ciudad, pero al mismo tiempo limita el proceso de negociación directo entre consumidores y productores. Esto muestra una mayor profesionalización de estos sistemas (solicitudes formales, comités de expertos, requisitos establecidos institucionalmente, etc.), aunque se establece un intermediario que se encarga de tareas supuestamente asumidas por el grupo de consumidores. Asimismo, a pesar de que existen rituales y actos para volver a conectar a los consumidores y a los productores, no todos los miembros de las CSA participan en estos procesos. Los horarios de trabajo de los miembros de estos grupos, la falta de un espacio permanente y la gran distancia a la que se encuentran las granjas afectan a la consolidación de estas prácticas. Todo esto, en cierta medida, puede llevar a cuestionar la legitimidad de la CSA como un sistema local transformador (Nost, 2014) y, al mismo tiempo, muestra la necesidad de explorar los procesos de reflexividad de los consumidores sobre su participación en estos sistemas. Sin embargo, las CSA siguen teniendo el potencial de reconectar al consumidor con el productor en cualquier momento, lo que es impensable en otros contextos, como en un supermercado. De acuerdo con Lamine (2005), el compromiso a largo o medio plazo de estos sistemas los constituye como una alternativa a las formas más convencionales de consumo de alimentos. 


\section{Referencias bibliográficas}

Adams, M. y Raisborough, J. (2008): «What can sociology say about fair trade?», Sociology, 42 (6), pp. 1165-1182.

Allen, P.; Fitzsimmons, M.; Goodman, M., y Warner, K. (2003): «Shifting plates in the agrifood landscape: the tectonics of alternative agrifood initiatives in California», Journal of Rural Studies, 19, pp. 61-75.

Alonso, L. E. (2005): «¿Un nuevo consumidor?», Ábaco, 2 (31), pp. 11-18.

Charmaz, K. (2006): Constructing grounded theory: a practical guide through qualitative analysis, Londres: Sage.

Coок, I. (2004): «Follow the thing. Papaya», Antipode, 36, pp. 642-664.

Corbin, J. y Strauss, A. (1990): «Grounded theory research: procedures, canons, and evaluative criteria», Qualitative Sociology, 13 (1), pp. 3-21.

Delgado Cabeza, M. (2010): «El sistema agroalimentario globalizado: imperios alimentarios y degradación social y ecológica», Revista de Economía Crítica, 10, pp. 32-61.

DeLind, L. B. (1999): «Close encounters with a CSA: the reflections of a bruised and somewhat wiser anthropologist», Agriculture and Human Values, 16, pp. 3-9.

Dowler, E.; Kneafsey, M.; Cox, R., y Holloway, L. (2009): «Doing food differently: reconnecting biological and social relationships through care for food», The Sociological Review, 57, pp. 200-221.

DuPuis, E. M. y Goodman D. (2005): «Should we go "home" to eat?: toward a reflexive politics of localism», Journal of Rural Studies, 21, pp. 359-371.

FeAGAN, R. (2007): «The place of food: mapping out the "local" in local food systems», Progress in Human Geography, 31, pp. 23-42.

Feagan, R. y Henderson, A. (2009): «Devon Acres CSA: local struggles in a global food system», Agriculture and Human Values, 26, pp. 203-217.

Feldmann, C. y Hamm, U. (2015): «Consumers' perceptions and preferences for local food: A review», Food Quality and Preference, 40, pp. 152-164.

Gertz, R. (2005): «Eco-labelling: a case for deregulation?», Law, Probability and Risk, 4, pp. 127-141.

Glaser, B. G. y Strauss, A. (1967): Discovery of grounded theory: strategies for qualitative research, Chicago: Aldine.

Heiskanen, E. (2005): «The performative nature of consumer research: consumers' environmental awareness as an example», Journal of Consumer Policy, 28 (2), pp. 179-201. 
Hinrichs, C. C. (2000): «Embeddedness and local food systems: notes on two types of direct agricultural markets», Journal of Rural Studies, 16, pp. 295303.

- (2003): «The practice and politics of food system localization», Journal of Rural Studies, 19, pp. 33-45.

JAROSz, L. (2008): «The city in the countryः growing alternative food networks in metropolitan areas», Journal of Rural Studies, 24, pp. 231-244.

Johnston, J. (2008): «Counter-hegemony or bourgeois piggery? Food politics and the case of FoodShare», en W. Wright y G. Middendorf (coords.) (2008): The fight over food, Pensilvania: The Pennsylvania State University Press, pp. 93-120.

KHAN, F. y PrIOR, C. (2010)ः «Evaluating the urban consumer with regard to sourcing local food: a heart of England study», International Journal of Consumer Studies, 34, pp. 161-168.

Kneafsey, M.; Cox, R.; Holloway, L.; Dowler, E.; Venn, L., y Tuomainen, H. (2008): Reconnecting consumers, producers and food: exploring alternatives, Oxford: Berg.

LAmine, C. (2005): «Settling shared uncertainties: local partnerships between producers and consumers», Sociologia Ruralis, 45, pp. 324-345.

Langreo Navarro, A. (2008): «El sistema alimentario mundial. Principales tendencias y efectos sobre los sistemas alimentarios locales», Revista Distribución y Consumo, 100, pp. 258-274.

Mauthner, N. y Doucet, A. (2003): «Reflexive accounts and accounts of reflexivity in qualitative data analysis», Sociology, 37 (3), pp. 413-431.

Micheletti, M.y Stolle, D. (2012): «Sustainable citizenship and new politics of consumption», Annals of the American Academy of Political and Social Science, 644, pp. 88-120.

Montagut, X. y Vivas, E. (coords.) (2007): Supermercados, no gracias. Grandes cadenas de distribución, impactos y alternativas, Barcelona: Icaria Editorial.

Murdoch, J. (2000): «Networks - a new paradigm of rural development?», Journal of Rural Studies, 16, pp. 407-419.

Nie, C. y Zepeda L. (2011): «Lifestyle segmentation of US food shoppers to examine organic and local food consumption», Appetite, 57, pp. 28-37.

Nost, E. (2014): «Scaling-up local foods: commodity practice in community supported agriculture (CSA)», Journal of Rural Studies, 34, pp. 152-160.

O'Neill, C. (2014)ः «Situating the "alternative" within the "conventional" - local food experiences from the East Riding of Yorkshire, UK», Journal of Rural Studies, 35, pp. 112-122. 
Osтrom, M. (2007): «The contribution of community supported agriculture (CSA) to movements for change in the agrifood system», en C. Hinrichs y T. Lyson (coords.): Remaking the North American food system, Nebraska: University of Nebraska Press, pp. 99-120.

Patton, M. Q. (2002): Qualitative research and evaluation methods, Thousand Oaks: Sage.

Perez, J.; Allen, P., y Brown, M. (2003): Community supported agriculture on the Central Coast: the CSA member experience, The Center for Agroecology and Sustainable Food Systems, Research Brief, California: University of California.

Pratt, J. (2007): «Food values. The local and the authentic», Critique of Anthropology, 27, pp. 285-300.

Reckwitz, A. (2002): «Toward a theory of social practices: a development in culturalist theorizing», European Journal of Social Theory, 5 (2), pp. 243-63.

Rudy, K. (2012): «Locavores, feminism, and the question of meat», The Journal of American Culture, 35, pp. 26-36.

Selfa, T. y Qazi, J. (2005): «Place, taste, or face-to-face? Understanding producer-consumer networks in "local" food systems in Washington State», Agriculture and Human Values, 22, pp. 451-464.

Seyfang, G. (2006): «Ecological citizenship and sustainable consumption: examining local food organic networks», Journal of Rural Studies, 22, pp. 383-395.

Thompson, C.; Pollio, H., y Locander, W. (1994): «The spoken and the unspoken: a hermeneutic approach to understanding the cultural viewpoints that underlie consumers' expressed meanings», Journal of Consumer Research, 21, pp. 432-451.

Thompson, C. y Coskuner-Balli, G. (2007): «Enchanting ethical consumerism. The case of community supported agriculture», Journal of Consumer Culture, 7, pp. 275-303.

Varul, M. (2008): «Consuming the campesino», Cultural Studies, 22, pp. 654679.

WARDE, A. (2005): «Consumption and theories of practice», Journal of Consumer Culture, 5 (2), pp. 131-153. 